\title{
Methamphetamine and Viagra Use: Relationship to Sexual Risk Behaviors
}

\author{
Dennis G. Fisher · Grace L. Reynolds • \\ Michael R. Ware · Lucy E. Napper
}

Received: 29 May 2008 / Revised: 9 February 2009/ Accepted: 16 February 2009/Published online: 28 March 2009

(C) The Author(s) 2009. This article is published with open access at Springerlink.com

\begin{abstract}
Recent studies show that Viagra and methamphetamine use are associated with unprotected anal intercourse among men who have sex with men (MSM). In Long Beach, California, we have reported on an association between Viagra use and the use of amphetamines during sex. The current research investigated the use of both Viagra and amphetamine in men in Long Beach, California. Data on 1,839 men recruited into HIV prevention and testing programs were collected using the Risk Behavior Assessment. A generalized logit model was constructed comparing ever having used both amphetamine and Viagra together and separately, as compared to never having used either (referent). Men who used both methamphetamine and Viagra showed a significantly higher prevalence of hepatitis B, syphilis, and HIV compared to those who used only one or neither drug. Of the 1,794 complete cases, $11.1 \%$ (199/1794) had used both amphetamine and Viagra. Of 20 potential risk and protective factors for use of amphetamine and Viagra, 12 were significant predictors: ever used gamma-hydroxybutyrate (GHB), ever used cocaine, ever used ecstasy, being infected with HIV, race = White compared to other, ever having hepatitis $\mathrm{B}$, ever using crack, ever given money to have sex, living in a hotel, ever been in drug treatment, and ever using heroin. The protective factor was being heterosexual. Viagra use was associated with insertive, and methamphetamine was associated with receptive, anal intercourse. GHB use appears to play a more important role than previously thought.
\end{abstract}

D. G. Fisher ( $\varangle)$ · G. L. Reynolds · M. R. Ware · L. E. Napper Center for Behavioral Research and Services, California State University, 1090 Atlantic Avenue, Long Beach, CA 90813, USA e-mail: dfisher@csulb.edu
Keywords Methamphetamine $\cdot$ Viagra $\cdot$ Drug abuse . Anal intercourse $\cdot$ Sexual risk $\cdot$ HIV

\section{Introduction}

Polydrug use that combines methamphetamine with other drugs has been documented in several studies (Halkitis \& Green, 2007; Patterson, Semple, Zians, \& Strathdee, 2005; Rawstorne, Digiusto, Worth, \& Zablotska, 2007). These studies have tended to focus on street drugs, such as ecstasy, cocaine, heroin and Ketamine. Recently, however, researchers have begun to report on the concurrent use of methamphetamine and Viagra ${ }^{\circledR}$ (sildenafil citrate) prior to and during sexual encounters (e.g., Mansergh et al., 2006; Semple, Zians, Strathdee, \& Patterson, 2008). These studies indicate that while methamphetamine and Viagra are both independently associated with high-risk sexual behavior, the behavioral risks and associated outcomes are significantly greater for users who combine both drugs. Spindler et al. (2007) reported that the prevalence of unprotected insertive anal sex with a male partner of unknown or different HIV status within a sample of men who have sex with men (MSM) was $19.1 \%$ for users of Viagra alone and $22.6 \%$ for users of methamphetamine alone. Prevalence rates jumped to $46.9 \%$ when methamphetamine was combined with Viagra. The increasing popularity of the combined use of methamphetamine and Viagra suggests that use of this sex-drug cocktail is worthy of a broader consideration, as it may identify a subgroup of individuals who are at even greater risk of HIV and sexually transmitted disease (STD) acquisition than those who use methamphetamine or Viagra alone or neither drug.

According to the World Health Organization, illicit use of methamphetamine among all drug use is second only to cannabis (Rawson, Anglin, \& Ling, 2002). MSM who use 
methamphetamine consistently report engaging in risky sexual behavior, including unprotected insertive and receptive anal sex (Crosby \& Mettey, 2004; Halkitis, Mukherjee, \& Palamar, 2007; Mansergh et al., 2001; Patterson \& Semple, 2003; Schwarcz et al., 2007) and several reports indicate that these users are at increased risk for acquisition of HIV and other STDs (Patterson \& Semple, 2003; Schwarcz et al., 2007; Semple, Zians, Grant, \& Patterson, 2006). Within Los Angeles county, MSM who use crystal methamphetamine are five times more likely to test positive for HIV than MSM who do not use the drug (Rosenblatt, 2007). Although MSM may be at increased risk for HIV infection compared to heterosexuals, risky sexual behavior is not exclusive to MSM. Research indicates that adolescent and adult heterosexual users of methamphetamine also report having multiple sex partners and engaging in unprotected sex while under the influence (Molitor, Truax, Ruiz, \& Sun, 1998).

Although originally developed as a medical aid to combat erectile dysfunction, recreational use of Viagra is not uncommon (Halkitis, Mukherjee et al., 2007; Mansergh et al., 2001). Like methamphetamine, recreational use of Viagra is strongly associated with risky sexual behavior and HIV (Crosby \& DiClemente, 2004; Fisher et al., 2006; Swearingen \& Klausner, 2005). Independent examinations of the behavioral risks associated with methamphetamine and the risks associated with recreational use of Viagra have found a dissimilar pattern of behavioral associations. For example, while the recreational use of Viagra has been found to be associated with unprotected insertive anal intercourse (UIA), reports on methamphetamine use by MSM have found use of the drug to be associated with unprotected receptive anal intercourse (URA) (Schwarcz et al., 2007).

These findings highlight an important distinction with respect to methamphetamine and Viagra use. Previous reports have identified methamphetamine use by MSM for the purpose of mood and energy to counteract the negative affect associated with HIV+ serostatus (Semple, Patterson, \& Grant, 2002) or within the context of raves and circuit parties (Halkitis, Parsons, \& Stirratt, 2001; Lee, Galanter, Dermatis, \& McDowell, 2003; Ross, Mattison, \& Franklin, 2003). Within these contexts, the use of methamphetamine may be incidental to sex (Ross et al., 2003). However, the linking of methamphetamine use with Viagra clearly places methamphetamine use strictly within the context of sex. This connection was previously noted in a study on Viagra use (Fisher et al., 2006) which found that the combination of methamphetamine and Viagra was reportedly used both before and during sex. This was further supported by reports suggesting that the recreational use of Viagra was associated with risky sexual behavior and not used solely for the purpose of enhancing sexual performance (Colfax et al., 2001).

The present study examined the combined use of methamphetamine and Viagra within a sample of men living in the
Long Beach, California area with the following specific aims: (1) to examine the prevalence of methamphetamine and Viagra use men in Long Beach-South Bay, California; (2) to examine the incremental demographic and behavioral correlates of methamphetamine and Viagra use; and (3) to characterize the infectious disease risk of the combined use of methamphetamine and Viagra. Using "no use of either drug" as the reference category, we constructed a multinomial logit model and compared behavioral risks associated with using methamphetamine alone, Viagra alone, and both drugs combined.

\section{Method}

Participants

Between May 2001 and July 2007, data were collected on 1,839 men between the age of 15 and 88 years $(M=40.66$ years, $S D=11.27$ ) residing in the Long Beach, California area. Participants were recruited through three HIV prevention and/or HIV/STD testing programs operated by the Center for Behavioral Research and Services at California State University, Long Beach. One program provided HIV/ AIDS prevention counseling for gay and bisexual men, one provided HIV/AIDS prevention counseling for illicit drug users and heavy alcohol users, and one provided HIV, STD, and hepatitis testing for a variety of behavioral risk groups. It was also possible for any client to use more than one program if they qualified. Sample demographics included $44.7 \%$ White, $24.5 \%$ Black, $21.3 \%$ Hispanic, and $9.7 \%$ other. Of the total sample, $47.5 \%$ identified as men who have sex with men (MSM)/men who have sex with men and women (MSMW) and $52.5 \%$ identified as heterosexual. HIV positive prevalence was $7.0 \%$ of those tested in the total sample.

\section{Measures}

\section{Risk Behavior Assessment (RBA)}

The RBA is a $20-30$ min, structured interview developed by the Community Research Branch of the National Institute on Drug Abuse (NIDA) in collaboration with the NIDA Cooperative Agreement for Community-Based Outreach/Intervention Research grantees. The RBA is administered by trained interviewers and asks questions related to HIV risk behaviors (e.g., drug use, needle sharing, and sexual behaviors), drug history, health history and status, work status, and income. Specific drug use variables include lifetime use or nonuse, age of first use, frequency of use during the most recent 30 day period (i.e., number of times used and number of days used). These assessments were made for alcohol, marijuana, cocaine (crack, powdered, and injection), heroin, speedball (heroin and 
cocaine mixed together), nonprescription methadone, other opiates, and amphetamine. Sexual risk behaviors included number of sexual partners during the most recent 30 day period, frequency and type of sexual activity, use of condom or barrier protection, and whether sex had been traded for drugs and/or money. The test-retest reliability of most of the questions and 48-h validity of the drug use variables have been found to be acceptable to very good (Dowling-Guyer et al., 1994; Fisher, Kuhrt-Hunstiger, Orr, \& Davis, 1999; Fisher, Reynolds, Jaffe, \& Johnson, 2007; Needle et al., 1995).

\section{Designer Drug Trailer (DDT)}

The DDT is an addendum to the RBA and assesses use of designer drugs. The format for the DDT was similar to the RBA and included questions about lifetime use and nonuse, age of first use, and frequency of use during the most recent 30-day period (i.e., number of times used and number of days used). Drugs assessed included methylene-dioxymethamphetamine (MDMA), Ketamine, gamma hydroxybutyrate/ gamma-butyrolactone (GHB/GHL), 2C-T-7, 2C-B, 5-MeODIPT (Foxy or Foxy methoxy), 2-MTA, Rohypnol, and Viagra.

\section{Procedure}

All participants were interviewed in individual sessions. At the beginning of each session, participants provided informed consent and were allowed to ask questions following protocols approved by the Institutional Review Board at California State University, Long Beach. Interview sessions lasted approximately $1.5 \mathrm{~h}$, during which time each participant was administered the RBA and DDT and completed several self-administered questionnaires. The conclusion of each session varied by program; however, all participants were given incentives for participation as well as referrals to other services as needed. All data collected for this project were protected by certificates of confidentiality.

\section{Statistical Analysis}

The data were analyzed using a multinomial logit model, sometimes referred to as a generalized logit model (Allison, 1999; Hosmer \& Lemeshow, 2000; McFadden, 1973). The data were analyzed in SAS 9.1.3 service pack 4. Because of missing values on one or more variables, only $1794(97.55 \%)$ of the data were used. There were four classifications of the data: those who did not use either drug $(n=762)$ was the referent group. The referent group was compared to: (1) those who used methamphetamine only ( $n=715)$, (2) those who used Viagra only ( $n=118$ ), and (3) those who used both methamphetamine and Viagra $(n=199)$.

\section{Results}

The proportion of those using methamphetamine immediately before or during sex in the methamphetamine-only group was lower $(24 \%)$ than in the group using both methamphetamine and Viagra, where it was $33 \% \chi^{2}(1, N=542)=3.83, p=$ .0502 . This demonstrated that the use of methamphetamine was more closely associated with sexual activity for those who used both methamphetamine and Viagra.

Table 1 shows the percentage of participants in each drug group who tested positive to six different laboratory tests. The four tests for sexually transmitted infections (hepatitis B core antigen, HIV, and two tests for syphilis (TP-PA and RPR) all showed significant differences in proportions and the drug group with the highest percentage for three of these four tests was the group using both methamphetamine and Viagra.

Table 2 shows the results of the generalized logit model comparing those who only used methamphetamine to the referent group, which was those who did not use either drug. The strongest risk factors were use of GHB/GHL and use of cocaine. Other drug risk factors were use of crack, ecstasy, and heroin. The methamphetamine-only group was also more likely to report being positive for HIV. Table 3 shows the results of the generalized logit model comparing those who only used Viagra to those who did not use either drug. Again, the strongest risk factors were use of GHB/GHL and cocaine. There was more emphasis on sex risk and less on drug risk in this group, so the risk factors of ever given money to have sex and the number of people had sex with distinguished the Viagra-only group from the referent group, but crack, ecstasy, and heroin were not significant risk factors for the Viagra only group. Being HIV positive was not significant.

Table 4 shows the generalized logit model comparing those who used both methamphetamine and Viagra to those who did not use either drug. The major drug risk factors were use of GHB/GHL, cocaine, and ecstasy, in addition to crack

Table 1 Methamphetamine and Viagra group by percent positive on laboratory test result

\begin{tabular}{llllllll}
\hline Lab test & $\begin{array}{l}\text { Neither } \\
\text { drug }\end{array}$ & $\begin{array}{l}\text { Meth } \\
\text { only }\end{array}$ & $\begin{array}{l}\text { Viagra } \\
\text { only }\end{array}$ & Both & $\chi^{2}$ & $n$ & $p$ \\
\hline Hepatitis A & 47.8 & 45.1 & 44.0 & 41.5 & 1.27 & 649 & ns \\
Hepatitis B & $13.0_{\mathrm{abc}}$ & $22.8_{\mathrm{b}}$ & $26.5_{\mathrm{c}}$ & $31.7_{\mathrm{a}}$ & 18.5 & 631 & .0003 \\
Hepatitis C & 64.0 & 61.0 & 66.7 & 44.1 & 3.51 & 157 & $\mathrm{~ns}$ \\
HIV & $3.8_{\mathrm{ab}}$ & $8.2_{\mathrm{b}}$ & 3.2 & $9.5_{\mathrm{a}}$ & 11.06 & 1021 & .0114 \\
TP-PA $^{\mathrm{a}}$ & $4.0_{\mathrm{abc}}$ & $8.8_{\mathrm{b}}$ & $15.6_{\mathrm{c}}$ & $15.3_{\mathrm{a}}$ & 16.1 & 580 & .0011 \\
RPR $^{\mathrm{b}}$ & $1.6_{\mathrm{ac}}$ & 4.4 & $6.7_{\mathrm{c}}$ & $8.3_{\mathrm{a}}$ & 9.5 & 582 & .0233 \\
\hline
\end{tabular}

Note: In all cases $d f=3$. Proportions in the same row that share the same subscript differ at $p<.05$ in pairwise $\chi^{2}$ comparisons

${ }^{a}$ Serodia TP-PA qualitative gelatin particle agglutination assay

b ASI rapid plasma reagin test 
Table 2 Generalized logit comparing those using methamphetamine only to those using neither drug

\begin{tabular}{llll}
\hline Variable & $B$ & $\begin{array}{l}\text { Odds } \\
\text { ratio }\end{array}$ & $95 \%$ CI \\
\hline Ever used GHB/GHL & 1.94 & 6.8 & $2.71,17.93$ \\
Ever use cocaine by itself & 1.66 & 5.3 & $3.90,7.08$ \\
Ever been told infected with HIV & 0.97 & 2.6 & $1.40,4.99$ \\
Ever use crack & 0.96 & 2.6 & $1.83,3.69$ \\
Are you living in a hotel & 0.93 & 2.5 & $1.52,4.21$ \\
Ever used ecstasy & 0.79 & 2.2 & $1.50,3.26$ \\
Ever been in drug treatment or detox & 0.73 & 2.1 & $1.49,2.89$ \\
White & 0.62 & 1.9 & $1.40,2.47$ \\
Ever use heroin & 0.52 & 1.7 & $1.19,2.38$ \\
\hline
\end{tabular}

Note: All odds ratios are significant if the $95 \%$ confidence interval does not include 1

Table 3 Generalized logit comparing those using Viagra only to those using neither drug

\begin{tabular}{llll}
\hline Variable & $B$ & $\begin{array}{l}\text { Odds } \\
\text { ratio }\end{array}$ & $95 \%$ CI \\
\hline Ever used GHB/GHL & 1.81 & 6.1 & $1.78,21.09$ \\
Ever use cocaine by itself & 0.75 & 2.1 & $1.31,3.44$ \\
White & 0.66 & 1.9 & $1.29,2.93$ \\
Ever given money to have sex & 0.62 & 1.9 & $1.09,3.17$ \\
How many people had sex with & 0.13 & 1.14 & $1.01,1.27$ \\
Do you consider yourself heterosexual & -0.95 & 0.4 & $0.22,0.69$
\end{tabular}

Note: All odds ratios are significant if the $95 \%$ confidence interval does not include 1

Table 4 Generalized logit comparing those using both methamphetamine and Viagra to those using neither drug

\begin{tabular}{lrrl}
\hline Variable & $B$ & $\begin{array}{c}\text { Odds } \\
\text { ratio }\end{array}$ & 95\% CI \\
\hline Ever used GHB/GHL & 2.38 & 10.8 & $4.30,28.80$ \\
Ever use cocaine by itself & 1.99 & 7.3 & $4.38,12.24$ \\
Ever used ecstasy & 1.84 & 6.3 & $3.89,10.29$ \\
Ever been told infected with HIV & 1.36 & 3.9 & $1.85,8.21$ \\
White & 1.13 & 3.1 & $2.07,4.65$ \\
Ever been told have hepatitis B & 1.12 & 3.1 & $1.69,5.61$ \\
Ever use crack & 1.07 & 2.9 & $1.73,4.92$ \\
Ever given money to have sex & 0.89 & 2.4 & $1.57,3.78$ \\
Are you living in a hotel & 0.82 & 2.3 & $1.17,4.41$ \\
Ever been in drug treatment or detox & 0.70 & 2.0 & $1.24,3.26$ \\
Ever use heroin & 0.56 & 1.7 & $1.05,2.88$ \\
Do you consider yourself heterosexual & -1.18 & 0.3 & $0.18,0.52$
\end{tabular}

Note: All odds ratios are significant if the $95 \%$ confidence interval does not include 1

and heroin. Both HIV and hepatitis B entered the model as risk factors and ever given money to have sex was also a risk factor. This was the only model that included hepatitis B.
Table 5 Drug use groups by sexual preference compared on anal intercourse type and mean frequency in 30 days

\begin{tabular}{lllll}
\hline Intercourse type & $\begin{array}{l}\text { Neither drug } \\
M(S D)\end{array}$ & $\begin{array}{l}\text { Meth only } \\
M(S D)\end{array}$ & $\begin{array}{l}\text { Viagra only } \\
M(S D)\end{array}$ & $\begin{array}{l}\text { Both } \\
M(S D)\end{array}$ \\
\hline Gay & & & & \\
Insertive anal & $1.7(4.26)_{\mathrm{a}}$ & $2.7(5.71)$ & $2.3(4.55)$ & $4.0(17.69)_{\mathrm{a}}$ \\
Receptive anal & $1.4(3.58)$ & $1.6(3.96)$ & $0.8(1.29)_{\mathrm{b}}$ & $2.1(5.41)_{\mathrm{b}}$ \\
Straight & & & & \\
Insertive anal & $0.5(2.07)$ & $0.4(1.82)$ & $0.4(1.88)$ & $0.9(3.72)$ \\
Receptive anal & $0.01(0.13)$ & $0.01(0.24)$ & $0.0(0.00)$ & $0.0(0.00)$ \\
\hline
\end{tabular}

Note: Frequencies of behavior for straight men were too low for meaningful statistical analysis. One-way ANOVA (gay only) for Drug Group on insertive anal sex $F(3,964)=2.98, p=.03$. One-way ANOVA (gay only) for Drug Group on receptive anal sex $F(3,964)=2.54$, $p=.055$. Means in the same row that share a subscript differ at $p<.05$ in the Tukey honestly significant difference comparison

In order to further explicate the model, the frequency of both insertive and receptive anal sex reported by both gay and heterosexual men was examined. Table 5 shows the means (separately for gay and straight) of the number of times participants reported having both receptive and insertive anal intercourse in the 30 days before interview. One-way ANOVAs of Drug use group on both receptive anal intercourse and insertive anal intercourse were performed for the gay men only because the frequencies of the behaviors were too low for the heterosexual men for meaningful statistical analysis. It is clear that, for the gay men, the group that used both methamphetamine and Viagra had the greatest mean number of both receptive and insertive anal intercourse. The significant comparison was between the group that did not use either drug and the group that used both methamphetamine and Viagra for insertive anal sex; the significant comparison for receptive anal sex was between the Viagra only group and the group that used both methamphetamine and Viagra. Correlation analysis assisted with further interpretation. For the gay men who used both drugs, there was a significant positive correlation between number of times used amphetamine immediately before or during sex and number of times had receptive anal intercourse $r(98)=.21$, $p=.04$. There was a non-significant inverse correlation between number of times used amphetamine immediately before or during sex and number of times had insertive anal intercourse $r(98)=-.01$.

\section{Discussion}

Data from a sample of men were used to construct a multinomial model (consisting of three separate comparisons) comparing those who used methamphetamine, Viagra, and both methamphetamine and Viagra, to a referent group of those who used neither methamphetamine nor Viagra. The 
major risk factor in all three comparison models was use of GHB, which was both strong and consistent. GHB was originally developed as an anesthetic (Freese, Miotto, \& Reback, 2002). An epidemic of GHB abuse started in the late 1980s, which our data suggest is still continuing (Nicholson \& Balster, 2001). Its use in conjunction with Viagra has been reported in New York (Halkitis \& Palamar, 2006; Halkitis, Palamar, \& Mukherjee, 2007), San Francisco (Colfax et al., 2001), Los Angeles, and Chicago (Carey et al., in press). We strongly agree that the recreational use of GHB has been understudied (Halkitis \& Palamar, 2006) as our findings point to a very strong association between the use of GHB and the use of both methamphetamine and Viagra.

Cocaine was also a strong risk factor that was consistent across the drug groups, although not as strong as GHB. This is not surprising in the model comparing methamphetamine use, given that they are both stimulants and that this model also included ecstasy, which is also a stimulant. Cocaine use has been found to be associated with unprotected anal intercourse in young MSM and the only drug associated in multivariate analysis with failure to use condoms (McNall \& Remafedi, 1999). A nationwide study found that cocaine use was associated with both unprotected receptive and insertive anal sex (Koblin et al., 2003). In a California study, cocaine use was associated with unprotected anal intercourse in both bivariate and multivariate analysis (Molitor, Facer, \& Ruiz, 1999). In our findings, cocaine use was the second strongest predictor in all three models. Even though our findings were in agreement with other published reports, this consistency in our top two predictors across our three models is noteworthy. Furthermore, there was a strong association between the models and the differences in prevalence of the infections.

Very few other reports have attempted to distinguish type of anal intercourse and associations with different drugs. Two reports that did make this attempt showed an association of methamphetamine with receptive anal intercourse that would agree with our findings (Mansergh et al., 2006; Rusch, Lampinen, Schilder, \& Hogg, 2004). For gay men, receptive anal intercourse was lowest in the Viagra only group. The gay men who used both methamphetamine and Viagra reported the highest mean frequency of both receptive and insertive anal intercourse. In addition to having more receptive anal intercourse, they also appeared to be having more insertive anal sex. Their higher amount of receptive anal sex was related to their use of methamphetamine as shown by the significant correlation between the use of amphetamine immediately before or during sex and the number of times had receptive anal intercourse. One report showed Viagra taken in combination with methamphetamine was associated with insertive anal intercourse (Spindler et al., 2007). We would argue that this effect was due to the Viagra and not the methamphetamine (Fisher et al., 2006; Mansergh et al., 2006).
Overall, non-White men were less likely to report using both methamphetamine and Viagra than White men. This finding was strongest in the comparison of those who used both drugs to those who used neither drug. The association between methamphetamine and anal intercourse, and Viagra and anal intercourse has been reported to be more common among White men (Carey et al., in press). Other researchers have also reported that Black men were less likely to use methamphetamine and Viagra (Halkitis \& Green, 2007). Number of sex partners only entered the model comparing those who used Viagra only to those who used neither drug. Ever having given money to have sex was also a factor in both the Viagra only comparison as well as the Viagra and methamphetamine comparison. The only protective factor was being heterosexual, that is, those who were heterosexual were less likely to be in the Viagra only group or the Viagra and methamphetamine group as compared to the group who used neither drug. This was not true for the methamphetamine only group comparison.

In conclusion, our data showed that those gay men who take both Viagra and methamphetamine engaged in higher levels of risky sex, and as a consequence, had a significantly higher prevalence of hepatitis B, untreated syphilis, and HIV. Although not significant, even the heterosexual men who took both drugs had higher frequencies of insertive anal sex. There have been calls for warning labels for Viagra as well as for scheduling Viagra as a controlled substance (Klausner \& Swearingen, 2004; Swearingen \& Klausner, 2005). Our data would suggest that Viagra by itself may not be the culprit, but rather the combination of methamphetamine and Viagra. This would suggest that methamphetamine abuse and/or dependence should be a contraindication in Viagra prescribing. GHB also probably plays a more important role than previously thought and needs to be investigated to a much greater extent. Future research should be careful to distinguish type of anal intercourse and which drug is associated with insertive as compared to receptive anal intercourse. Our findings support the notion that Viagra is associated with insertive, and methamphetamine is associated with receptive, anal intercourse. Men who take both Viagra and methamphetamine are at much higher risk for hepatitis B, HIV and untreated syphilis because they engage in much higher rates of anal intercourse than any of the comparison groups.

Acknowledgments This research was funded in part by contract numbers H700938 and H700939 from the Office of AIDS Programs and Policy, County of Los Angeles, California. It was also funded in part by contract number 30391 from the Department of Health and Social Services, City of Long Beach, California. Funding for Dr. Napper was provided in part by National Research Service Award 1 F32 DA02290201A1 from the National Institute on Drug Abuse.

Open Access This article is distributed under the terms of the Creative Commons Attribution Noncommercial License which permits any 
noncommercial use, distribution, and reproduction in any medium, provided the original author(s) and source are credited.

\section{References}

Allison, P. D. (1999). Logistic regression using the SAS system: Theory and application. Cary, NC: SAS Institute Inc.

Carey, J. W., Mejia, R., Bingham, T., Ciesielski, C., Gelaude, D., Herbst, J. H., et al. (in press). Drug use, high-risk sex behaviors, and increased risk for recent HIV infection among men who have sex with men in Chicago and Los Angeles. AIDS and Behavior. doi: 10.1007/s10461-008-9403-3

Colfax, G. N., Mansergh, G., Guzman, R., Vittinghoff, E., Marks, G., Rader, M., et al. (2001). Drug use and sexual risk behavior among gay and bisexual men who attend circuit parties: A venue-based comparison. Journal of Acquired Immune Deficiency Syndrome, 28, 373-379.

Crosby, R., \& DiClemente, R. J. (2004). Use of recreational Viagra among men having sex with men. Sexually Transmitted Infections, $80,466-468$

Crosby, R., \& Mettey, A. (2004). A descriptive analysis of HIV risk behavior among men having sex with men attending a large sex resort. Journal of Acquired Immune Deficiency Syndrome, 37, 1496-1499.

Dowling-Guyer, S., Johnson, M. E., Fisher, D. G., Needle, R., Watters, J., Andersen, M., et al. (1994). Reliability of drug users' selfreported HIV risk behaviors and validity of self-reported recent drug use. Assessment, 1, 95-102.

Fisher, D. G., Kuhrt-Hunstiger, T. I., Orr, S. M., \& Davis, D. C. (1999). Hepatitis B validity of drug users' self-report. Psychology of Addictive Behaviors, 13, 33-38.

Fisher, D. G., Marlow, R., Rosenberg, R., Reynolds, G. L., Ferell, N., \& Jaffe, A. (2006). Recreational Viagra use and sexual risk among drug abusing men. American Journal of Infectious Diseases, 2, 107-114.

Fisher, D. G., Reynolds, G. L., Jaffe, A., \& Johnson, M. E. (2007). Reliability, sensitivity and specificity of self-report of HIV test results. AIDS Care, 19, 692-696.

Freese, T. E., Miotto, K., \& Reback, C. J. (2002). The effects and consequences of selected club drugs. Journal of Substance Abuse Treatment, 23, 151-156.

Halkitis, P. N., \& Green, K. A. (2007). Sildenafil (Viagra) and club drug use in gay and bisexual men: The role of drug combinations and context. American Journal of Men's Health, 1, 139-147.

Halkitis, P. N., Mukherjee, P. P., \& Palamar, J. J. (2007). Multi-level modeling to explain methamphetamine use among gay and bisexual men. Addiction, 102(Suppl. 1), 76-83.

Halkitis, P. N., \& Palamar, J. J. (2006). GHB use among gay and bisexual men. Addictive Behaviors, 31, 2135-2139.

Halkitis, P. N., Palamar, J. J., \& Mukherjee, P. P. (2007). Poly-club-drug use among gay and bisexual men: A longitudinal analysis. Drug and Alcohol Dependence, 89, 153-160.

Halkitis, P. N., Parsons, J. T., \& Stirratt, M. J. (2001). A double epidemic: Crystal methamphetamine drug use in relation to HIV transmission among gay men. Journal of Homosexuality, 41(2), $17-35$.

Hosmer, D. W., \& Lemeshow, S. (2000). Applied logistic regression. New York: John Wiley.

Klausner, J., \& Swearingen, S. (2004). Petition to the Food and Drug Administration to change the labeling and take further action to address the association of Viagra (Sildenafil) use and increased STD transmission, including HIV infection. City and County of San Francisco: STD Prevention and Control Services.
Koblin, B. A., Chesney, M. A., Husnik, M. J., Bozeman, S., Celum, C. L., Buchbinder, S., et al. (2003). High-risk behaviors among men who have sex with men in 6 US cities: Baseline data from the EXPLORE study. American Journal of Public Health, 93, 926932.

Lee, S. J., Galanter, M., Dermatis, H., \& McDowell, D. (2003). Circuit parties and patterns of drug use in a subset of gay men. Journal of Addictive Diseases, 22, 47-60.

Mansergh, G., Colfax, G. N., Marks, G., Rader, M., Guzman, R., \& Buchbinder, S. (2001). The Circuit Party Men's Health Survey: Findings and implications for gay and bisexual men. American Journal of Public Health, 91, 953-958.

Mansergh, G., Shouse, R. L., Marks, G., Guzman, R., Rader, M., Buchbinder, S., et al. (2006). Methamphetamine and sildenafil (Viagra) use are linked to unprotected receptive and insertive anal sex, respectively, in a sample of men who have sex with men. Sexually Transmitted Infections, 82, 131-134.

McFadden, D. (1973). Conditional logit analysis of qualitative choice behavior. In P. Zarembka (Ed.), Frontiers in econometrics (pp. 105-142). New York: Academic Press.

McNall, M., \& Remafedi, G. (1999). Relationship of amphetamine and other substance use to unprotected intercourse among young men who have sex with men. Archives of Pediatrics and Adolescent Medicine, 153, 1130-1135.

Molitor, F., Facer, M., \& Ruiz, J. D. (1999). Safer sex communication and unsafe sexual behavior among young men who have sex with men in California. Archives of Sexual Behavior, 28, 335-343.

Molitor, F., Truax, S. R., Ruiz, J. D., \& Sun, R. K. (1998). Association of methamphetamine use during sex with risky sexual behaviors and HIV infection among non-injection drug users. Western Journal of Medicine, 168, 93-97.

Needle, R., Fisher, D. G., Weatherby, N., Chitwood, D., Brown, B., Cesari, H., et al. (1995). Reliability of self-reported HIV risk behaviors of drug users. Psychology of Addictive Behaviors, 9, 242-250.

Nicholson, K. L., \& Balster, R. L. (2001). GHB: A new and novel drug of abuse. Drug and Alcohol Dependence, 63, 1-22.

Patterson, T. L., \& Semple, S. J. (2003). Sexual risk reduction among HIV-positive drug-using men who have sex with men. Journal of Urban Health, 80(4 Suppl. 3: iii), 77-87.

Patterson, T. L., Semple, S. J., Zians, J. K., \& Strathdee, S. A. (2005). Methamphetamine-using HIV-positive men who have sex with men: Correlates of polydrug use. Journal of Urban Health, 97, 1067-1075.

Rawson, R. A., Anglin, M. D., \& Ling, W. (2002). Will the methamphetamine problem go away? Journal of Addictive Diseases, 21, 5-19.

Rawstorne, P., Digiusto, E., Worth, H., \& Zablotska, I. (2007). Associations between crystal methamphetamine use and potentially unsafe sexual activity among gay men in Australia. Archives of Sexual Behavior, 36, 646-654.

Rosenblatt, S. (2007, April 11). More gay men using meth, study finds: Use of the drug, which is associated with HIV transmission, has surged since 2005 , according to data collected by a nonprofit agency. Los Angeles Times.

Ross, M. W., Mattison, A. M., \& Franklin, D. R. (2003). Club drugs and sex on drugs are associated with different motivations for gay circuit party attendance in men. Substance Use and Misuse, 38, 1173-1183.

Rusch, M., Lampinen, T. M., Schilder, A., \& Hogg, R. S. (2004). Unprotected anal intercourse associated with recreational drug use among young men who have sex with men depends on partner type and intercourse role. Sexually Transmitted Diseases, 31, 492-498.

Schwarcz, S., Scheer, S., McFarland, W., Katz, M., Valleroy, L., Chen, S., et al. (2007). Prevalence of HIV infection and predictors of hightransmission sexual risk behaviors among men who have sex with men. American Journal of Public Health, 97, 1067-1075. 
Semple, S. J., Patterson, T. L., \& Grant, I. (2002). Motivations associated with methamphetamine use among HIV+ men who have sex with men. Journal of Substance Abuse Treatment, 22, 149-156.

Semple, S. J., Zians, J., Grant, I., \& Patterson, T. L. (2006). Sexual risk behavior of HIV-positive methamphetamine-using men who have sex with men: The role of partner serostatus and partner type. Archives of Sexual Behavior, 35, 461-471.

Semple, S. J., Zians, J., Strathdee, S. A., \& Patterson, T. L. (2008). Sexual marathons and methamphetamine use among HIV-positive men who have sex with men. Archives of Sexual Behavior. doi: 10.1007/s10508-10007-19292-y.

Spindler, H. H., Scheer, S., Chen, S. Y., Klausner, J. D., Katz, M. H., Valleroy, L. A., et al. (2007). Viagra, methamphetamine, and HIV risk: Results from a probability sample of MSM, San Francisco. Sexually Transmitted Diseases, 38, 586-591.

Swearingen, S. G., \& Klausner, J. D. (2005). Sildenafil use, sexual risk behavior, and risk for sexually transmitted diseases, including HIV infection. American Journal of Medicine, 118, 571-577. 